\title{
AMENDMENTS
}

\section{Publisher Correction: Seasonal plasticity and diel stability of H3K27me3 in natural fluctuating environments}

Haruki Nishio (D), Atsushi J. Nagano (D), Tasuku Ito, Yutaka Suzuki and Hiroshi Kudoh (D)

Correction to: Nature Plants https://doi.org/10.1038/s41477-020-00757-1, published online 31 August 2020.

In the version of this Letter originally published, the $y$-axis label in Fig. $4 \mathrm{~g}$ "H3K27me3 ( $\log _{2}$ [adjusted $P$ ])" was incorrect; it should have read "H3K27me3 ( $\log _{2}$ [adjusted rpkm])". All versions of the Letter have been amended.

Published online: 23 November 2020

https://doi.org/10.1038/s41477-020-00822-9

(c) The Author(s), under exclusive licence to Springer Nature Limited 2020 